\title{
Initiation of a critical pathway for pancreaticoduodenectomy at an academic institution - the first step in multi-disciplinary team building
}

\author{
Eugene P. Kennedy \\ Thomas Jefferson University \\ Ernest L. Rosato \\ Thomas Jefferson University \\ Patricia K. Sauter \\ Thomas Jefferson University \\ Laura M. Rosenberg \\ Fbliowathilefferssonditidikersititbrks at: https://jdc.jefferson.edu/surgeryfp \\ tabdod甲orieasurgery Commons \\ Themesiseffersos Wwiversitw access to this document benefits you
}

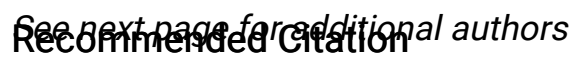

Kennedy, Eugene P.; Rosato, Ernest L.; Sauter, Patricia K.; Rosenberg, Laura M.; Doria, Cataldo; Marino, Ignazio R.; Chojnacki, Karen A.; Berger, Adam C.; and Yeo, Charles, "Initiation of a critical pathway for pancreaticoduodenectomy at an academic institution -- the first step in multidisciplinary team building" (2007). Department of Surgery Faculty Papers. Paper 4. https://jdc.jefferson.edu/surgeryfp/4

This Article is brought to you for free and open access by the Jefferson Digital Commons. The Jefferson Digital Commons is a service of Thomas Jefferson University's Center for Teaching and Learning (CTL). The Commons is a showcase for Jefferson books and journals, peer-reviewed scholarly publications, unique historical collections from the University archives, and teaching tools. The Jefferson Digital Commons allows researchers and interested readers anywhere in the world to learn about and keep up to date with Jefferson scholarship. This article has been accepted for inclusion in Department of Surgery Faculty Papers by an authorized administrator of the Jefferson Digital Commons. For more information, please contact: JeffersonDigitalCommons@jefferson.edu. 


\section{Authors}

Eugene P. Kennedy, Ernest L. Rosato, Patricia K. Sauter, Laura M. Rosenberg, Cataldo Doria, Ignazio R. Marino, Karen A. Chojnacki, Adam C. Berger, and Charles Yeo 


\title{
Initiation of a Critical Pathway for Pancreaticoduodenectomy at an Academic Institution - The First Step in Multi-Disciplinary Team Building
}

\author{
Eugene P. Kennedy, MD \\ Ernest L. Rosato, MD, FACS \\ Patricia K. Sauter, RN, ACNP \\ Laura M. Rosenberg, BS \\ Cataldo Doria, MD \\ Ignazio R. Marino, MD, FACS \\ Karen A. Chojnacki, MD, FACS \\ Adam C. Berger, MD, FACS \\ Charles J. Yeo, MD, FACS \\ Department of Surgery \\ Jefferson Medical College \\ Thomas Jefferson University \\ Philadelphia, PA
}

Correspondence:

Eugene P Kennedy, MD

1025 Walnut Street

Suite 605 College Building

Philadelphia, PA 19107

(215)955-9402

Fax (215) 923-6609

Eugene.Kennedy@Jefferson.edu

To be presented at the $118^{\text {th }}$ Annual meeting of the Southern Surgical Association; December 3-6, 2006; Palm Beach, Florida.

Running Header: Critical Pathway for PD

Key words: critical pathway; pancreaticoduodenectomy; length of stay; hospital charges. 


\begin{abstract}
Objective: This study was designed to identify quantifiable parameters to track performance improvements brought about by the implementation of a critical pathway for complex alimentary tract surgery.
\end{abstract}

Background: Pancreaticoduodenectomy (PD) is a complex general surgical procedure performed in varying numbers at many academic institutions. Originally associated with significant perioperative morbidity and mortality, multiple studies have now shown that this operation can be performed quite safely at high volume institutions that develop a particular expertise. Critical pathways are one of the key tools used to achieve consistently excellent outcomes as these institutions. It remains to be determined if implementation of a critical pathway at an academic institution with prior moderate experience with PD will result in performance gains and improved outcomes.

Methods: Between January 1, 2004 and October 15, 2006135 patients underwent PD, 44 before the implementation of a critical pathway on October 15, 2005, and 91 after. Perioperative and postoperative parameters were analyzed retrospectively to identify those that could be used to track performance improvement and outcomes. Key aspects of the pathway include spending the night of surgery in the intensive care unit with careful attention to fluid balance, early mobilization on post-operative day one, aggressive early removal of encumbrances such as nasogastric tubes and urinary catheters, early postoperative feeding, and targeting discharge for postoperative day 6 or 7 . 
Results: The pre- and post-pathway implementation groups were not statistically different with regards to age, sex, race, or pathology (malignant versus benign). Perioperative mortality, operative blood loss, and number of transfused units of packed red blood cells were also similar. As compared to the pre-pathway group, the postpathway group had a significantly shorter postoperative length of stay (13 versus 7 days, $\mathrm{P} \leq 0.0001$ ), operative time ( $435 \pm 14$ minutes versus $379 \pm 12$ minutes, $\mathrm{P} \leq 0.0001)$, and in room non-operative time ( $95 \pm 4$ minutes versus $76 \pm 2$ minutes, $\mathrm{P} \leq 0.0001)$. Total hospital charges were significantly reduced from $\$ 240,242 \pm \$ 32,490$ versus $\$ 126,566 \pm$ $\$ 4883(\mathrm{P} \leq 0.0001)$ after pathway implementation. Postoperative complication rates remained constant ( $44 \%$ pre-pathway versus $37 \%$ after, $\mathrm{P}=\mathrm{NS}$ ). Readmission rates were not negatively affected by the reduction in length of stay, with a $7 \%$ readmission rate prior to implementation and a $7.7 \%$ rate after implementation.

Conclusion: Implementation of a critical pathway for a complex procedure can be demonstrated to improve short-term outcomes at an academic institution. This improvement can be quantified and tracked and has implications for better utilization of resources (greater OR and hospital bed availability) and overall cost containment. With a very conservative estimate of 75 pancreaticoduodenectomies per year by this group, this translates to a savings of 450 hospital days and over $\$ 8,550,000$ in hospital charges on an annual basis. As we enter the "pay for performance" era, institutions will be required to generate such data in order to retain patient volumes, attract new patients, and receive "incentive payments" for high quality services rendered. 


\section{INTRODUCTION}

Healthcare providers are under increasing pressure to control costs while maintaining or improving outcomes. The implementation of critical pathways (or fasttracking protocols) is one approach to this challenge that has been applied to many different surgical procedures (1-10). Critical pathways are best described as structured multidisciplinary care plans that detail the essential steps in the care of patients with a specific clinical problem (11). They provide a timeline of the ideal sequence of treatment related events with daily goals, to assist care providers in administering care with optimal efficiency. Multiple reports have credited these pathways with improving efficiency, reducing length of hospital stay, and helping to control costs $(1,4,5,9)$.

Recently, several reviews have been published that question the true value of critical pathways (11-15). The criticisms contained in these articles include (1) the possibility that performance improvements are the result of patient selection bias, with only healthy patients "cherry picked" for inclusion in pathway treatment giving unfair advantage over unfiltered pre-pathway controls or, (2) that only pathway implementation with substantial improvements get reported (12). Additionally, the articles collectively cite a universal decrease in length of hospital stay as a secular trend across all of medicine, for which pathways are being given unfair credit (14).

Recognizing these issues, we evaluated the impact of introducing a critical pathway for a complex general surgical procedure (pancreaticoduodenectomy) at a major academic institution. Pancreaticoduodenectomy is a procedure performed in varying numbers at many academic institutions. Originally associated with significant perioperative morbidity and mortality, multiple studies have now shown that this 
operation can be performed quite safely at high volume institutions that develop a particular expertise (16). Critical pathways have been cited as one of the key tools used to achieve consistently excellent outcomes as these institutions. The implementation of the pathway for pancreaticoduodenectomy was timed to coincide with an expected rise in case volume. The aim of this study was to determine if implementation of a critical pathway at an academic institution with prior moderate experience with PD would result in performance gains and improved outcomes.

\section{METHODS}

Patients

The records of 135 consecutive patients undergoing pancreaticoduodenectomy at Thomas Jefferson University Hospital from January 1, 2004 through October 15, 2006 were reviewed. A critical pathway for pancreaticoduodenectomy was implemented on October 15, 2005 at the time of the arrival of a new Chair of Surgery (C.J.Y.) and after the recruitment of a team focused on pancreatic surgery (E.P.K. and P.K.S.). Additional critical pathways for distal pancreatectomy and palliative double bypass (gastrojejunostomy and hepaticojejunostomy) were similarly implemented, but are not part of this report. Fourty-four patients treated prior to the implementation of the pathway were compared to 91 patients treated after pathway implementation. Patients undergoing total pancreatectomy and right-sided completion pancreatectomy were also included, as they were treated according to pathway standards. Patients with both malignant and benign disease were included in this analysis. All patients undergoing pancreaticoduodenectomy after pathway implementation were treated according to 
pathway standards and are included in this analysis. Pathway implementation included numerous formal educational sessions with anesthesia, nursing, nutrition, and surgical house staff. Furthermore, pathway implementation included formal initiation of computerized standard order sets as part of the Thomas Jefferson University Hospital computerized order entry system.

Components of the critical pathway

The pathway utilized was previously developed and utilized at a high volume institution (Johns Hopkins Medical Institution) by two of the participating surgeons (C.J.Y. and E.P.K.) and by an experienced clinical nurse practitioner (P.K.S.). The pathway outlines the daily progress made by a patient without postoperative complications after surgery. (Table 1) Expectations with respect to all aspects of care are outlined for each postoperative day. Templates were generated for standardized order sets in the hospital computerized order entry system.

Pathway execution begins at the pre-operative office visit, with education of patients and families about general pathway goals and expectations, including the targeting of discharge for postoperative day 6 or 7 . All patients are instructed to ingest a mild mechanical bowel prep at home the night before surgery and patients are admitted as same day surgery patients. Consents for operation and research studies are obtained in advance of the day of surgery. Standardized orders for perioperative antibiotics and deep venous thrombosis (DVT) prophylaxis (subcutaneous heparin and thromboembolic deterrent (TED) stockings) are utilized in the preoperative holding area. In the operating room, patients are routinely monitored, usually with a radial arterial catheter. Sequential 
compression devices (SCDs) are utilized. Central venous access is obtained only when deemed necessary by the attending anesthesiologist. Epidural analgesia is not utilized. A nasogastric tube is placed after induction of anesthesia and two closed suction drains are placed during surgery.

Patients are extubated in the operating room when no contraindication exists and spend the night of surgery in an intensive care setting. Electrolyte abnormalities and fluid status are aggressively monitored and corrected. Postoperative analgesia is provided with intravenous narcotics via a patient controlled anesthesia (PCA) device. All patients also receive an intravenous proton pump inhibitor (PPI) and a beta-blocker, in addition to subcutaneous heparin and 24 hours of prophylactic antibiotic coverage.

Patients are mobilized in the early morning of the first postoperative day. The nasogastric tube is removed that morning and patients are started on sips of water and ice chips ( $\leq 30$ cc per hour). SCDs are discontinued, while TED stockings, subcutaneous heparin, intravenous beta-blockade, and PPI are continued until hospital discharge. Patients are transferred to the floor and ambulated with the assistance of staff.

On postoperative day two, patients are advanced to an unlimited clear liquid diet. The urinary catheter is removed and patients are assisted in increasing their frequency and duration of ambulation. Fluids are minimized and most patients receive low dose diuretics to aid in the mobilization of the perioperative fluid which was administered intraoperatively and immediately postoperatively.

In most patients the initiation of a regular diet begins on postoperative day three, with oral pancreatic enzyme supplementation. Medications, including beta-blockade and PPI, are continued as intravenous formulations until postoperative day four to assure that 
a diet is tolerated. Intravenous fluids are discontinued on postoperative day four. The surgical drains (typically two) are removed sequentially on postoperative days four and five, if appropriate. Medical oncology and radiation oncology are consulted when appropriate on postoperative day five. Pre-printed discharge instructions are distributed to allow time for patients and their families to review and formulate questions prior to discharge.

On postoperative days six and seven, patients continue to increase activity levels and arrangements for discharge are made. Discharge is targeted for postoperative day six or seven. A follow-up appointment is scheduled for four weeks after discharge. Typical discharge medications include necessary preoperative medications plus a PPI, pancreatic enzymes, and analgesics (typically an oxycodone containing oral preparation).

\section{Patient outcomes}

Data collected for analysis included demographics, diagnosis, operative blood loss and blood transfusions, length of operation, length of non-operative time in operating room, perioperative complication rates, perioperative mortality, length of postoperative hospital stay, total hospital charges, and readmission rate. Data were obtained from retrospective chart review for patients treated prior to implementation of the critical pathway and from a prospective clinical data base for patients treated after implementation. Common postoperative complications analyzed included pancreatic fistula (defined as output of more than $30 \mathrm{ml}$ per day of amylase rich fluid (more than 3 times serum value) for greater than 10 days postoperatively), delayed gastric emptying (DGE) (defined as persistent vomiting or inability to tolerate diet requiring replacement 
of nasogastric tube), and wound infection (defined by standard clinical criteria and requiring intervention). Perioperative mortality was defined as death within 30 days of surgery. Readmission rate was calculated based upon readmission to Thomas Jefferson University Hospital within 30 days of discharge. Length of operation was obtained from a query of the Thomas Jefferson University Hospital operating room information

management system. Financial data were provided by JeffCare, Inc., the Jefferson Health System’s Physician Hospital Organization.

Statistical analyses

Data analyses to determine level of significance of differences in characteristics and outcomes between the two groups of patients were performed using a 2 -sample $t$ test or Fisher's exact test, where appropriate. Data are expressed as mean \pm standard error where applicable or as a percentage where noted. Data on length of stay are presented as a median. Statistical significance was considered to have been achieved at the $p \leq 0.05$ level.

\section{RESULTS}

Demographics (Table 2)

The pre-pathway and post-pathway groups were similar with respect to the analyzed demographic data. Median age (60 years pre-pathway versus 66 years postpathway) was not significantly different between the groups. Similarly, distribution by sex (47\% female pre-pathway versus 55\% female post-pathway) and race ( $79 \%$ white, 12\% African American, 9\% other pre-pathway versus 87\% white, 5\% African American, 
$8 \%$ other post-pathway) was also comparable between the groups. The pathology in the resection specimen was also similar with $74 \%$ of the pre-pathway patients undergoing resection for malignant disease, compared to $70 \%$ of the post-pathway patients.

Intraoperative Parameters (Table 2)

Several intraoperative parameters were assessed. Operative blood loss $(549 \pm 48$ $\mathrm{ml}$ pre-pathway versus $646 \pm 29 \mathrm{ml}$ post-pathway) and units of packed red blood cells transfused during surgery $(0.72 \pm 0.16$ units pre-pathway versus $0.64 \pm 0.13$ units postpathway) did not significantly change with the implementation of the critical pathway. The length of operation as well as the length of time patients spend in the operating room did change significantly, however. Operative length (from incision to closure) decreased from $435 \pm 14$ minutes pre-pathway to $379 \pm 12$ minutes post-pathway $(\mathrm{P} \leq 0.0001)$. Non-operative time spent in the operating room (defined as the time from entry into room until incision plus the time from closure until room departure) fell from $95 \pm 4$ minutes pre-pathway to $76 \pm 2$ minutes post-pathway $(\mathrm{P} \leq 0.0001)$.

\section{Post-operative parameters (Table 3)}

The perioperative complication rate for pancreaticoduodenectomy did not change significantly with the implementation of the critical pathway. The overall perioperative complication rate was $44 \%$ prior to the implementation of the critical pathway and $37 \%$ afterwards. Rates for specific complications including pancreatic fistula (9\% pre-pathway versus $2 \%$ post-pathway), DGE ( $7 \%$ pre-pathway versus $8 \%$ post-pathway), and wound infection ( $9 \%$ pre-pathway versus $13 \%$ post-pathway) were similar as well. Other less 
common complications including atrial fibrillation and other cardiovascular events, pneumonia, intra-abdominal abscess (not containing amylase rich fluid) requiring interventional radiology drainage, small bowel obstruction, and deep venous thrombosis were also equally distributed between the groups (25\% versus $20 \%$ ). The 30 day mortality rate before and after pathway implementation was unchanged, at $2.3 \%$ prepathway compared to $1.1 \%$ post-pathway. Of note, postoperative length of hospital stay was significantly shortened by utilization of the critical pathway. Prior to pathway implementation the median length of hospital stay was 13 days. Implementation of the pathway resulted in a reduction of length of stay to 7 days $(\mathrm{P} \leq 0.0001)$. This paralleled a reduction in total hospital charges from $\$ 240,242 \pm \$ 32,490$ pre-pathway to $\$ 126,566 \pm$ $\$ 4883$ post-pathway $(\mathrm{P} \leq 0.0001)$. The reduction in length of hospital stay did not result in an increase in hospital readmissions, with 30 day readmission rates being $7 \%$ for prepathway patients and $7.7 \%$ for post-pathway patients.

\section{DISCUSSION}

The provision of high quality, cost-effective health care is a goal shared by all health care providers. As we approach the "pay-for-performance" era, strategies that maintain or improve quality outcomes while increasing the efficient use of limited resources gain increasing value. Critical pathways have been adopted at many institutions as a means to promote quality and efficient care. However, there are detractors who question the value of critical pathways. 
A number of studies have been published that question the value of critical pathways (11-14). These critiques revolve around three main points. First, critics claim that pathways are simply superimposed upon underlying, pre-existing trends. The reductions in length of stay cited by critical pathway authors as proof of efficacy are attributed, by critics, to overall trends in healthcare and outside economic pressures targeting shorter lengths of stay. Critics note that lengths of stay were declining prior to pathway implementation and that rates of decline were not increased by most pathways (13). Secondly, the selective application of pathways in some reports (4) is used by critics as evidence that the performance improvements attributed to critical pathways are simply the result of patient selection bias (12). Critics claim, accurately, that if healthier patients are placed on critical pathways, while patients with greater co-morbidities are placed in non-pathway control groups, then outcomes will invariably improve, independent of the efficacy of the pathway itself. Thirdly, critics claim that pathways require substantial resources to develop, implement and maintain (14). They claim that critical pathways depend on local processes and organizational structure requiring the development of unique pathways for each institution (13). The costs associated with this development have been alleged to offset the economic benefits of pathway implementation.

This study was designed to assess the impact of implementing a critical pathway at a major academic institution. The pathway was implemented fully formed, timed to coincide with a planned significant increase in operative volume and was introduced to all health care professionals at Thomas Jefferson University Hospital to allow comment and "buy-in." Its implementation was acute, as opposed to a gradual phase in over weeks or months. The hypothesis was that by using a critical pathway, a large institution with 
moderate experience with a particular complex procedure could significantly increase operative volume, while maintaining or improving measurable quality associated outcomes.

Several observations can be drawn from our results. The similarity in demographics argues against selection bias being the basis for the performance improvements described. Concerning measured intraoperative parameters, pathway implementation did not impact factors that can be considered surgeon dependant, such as blood loss or total units of packed red cells transfused. It did improve parameters that can be classified as system or multi-disciplinary team based, such as non-operative time in the room. This can be explained by such pathway elements as standardized central venous access and preoperative management and standardization of postoperative patient destination, eliminating delays associated with queries about the need for a monitored or ICU bed.

Postoperative parameter improvements that can be linked to pathway implementation start with postoperative length of hospital stay. Multiple factors contributed to the reduction in length, from altering patient expectations to education of hospital healthcare providers to earlier mobilization and return of oral intake. This reduction in length of postoperative hospital stay correlates directly with a significant reduction in total hospital charges. The shortening of postoperative hospital stay did not come at the expense of increased perioperative complications, mortality, or readmission rates, all of which were already at or above national standards.

As this study illustrates, a critical pathway can be successfully implemented at an academic medical center, prompting a significant decrease in length of postoperative 
hospital stay, total hospital charges, and operative times. This can be done in conjunction with a significant increase in surgical volume. Components of the critical pathway concept from documented goals, to increased awareness, to education of the healthcare team all contribute to the efficacy of critical pathways as performance improvement tools. When the above mentioned common criticisms of critical pathways are applied, the implementation of this pathway is still found to be efficacious. As concerns critical pathways taking credit for underlying trends in length of stay, the length of stay for pancreaticoduodenectomy had only deceased from a median of 15 days to 13 days at Thomas Jefferson University Hospital over the four years prior to the period covered by this study. Clearly, the rate of decrease in length of stay was impacted by implementation of this critical pathway. Further, selection bias was limited in this study. All patients undergoing pancreaticoduodenectomy at Thomas Jefferson University Hospital were treated on the newly introduced clinical pathway and are included in this report.

Of note, the costs of implementing this pathway were minimal. The pathway utilized was brought (with minor modifications) to Thomas Jefferson University Hospital from another institution at which three of the authors had extensive experience. It was successfully implemented without requiring substantial resources. Furthermore, its importation argues against the concept that critical pathways must be developed uniquely for each institution choosing to utilize them. The ability to transfer effective pathways from one institution to another should result in easier implementation, without significant expense. 
Critical pathways are an effective tool for quality improvement and cost containment. In appropriate settings, they can be implemented quickly and with limited expenditure of resources. Making performance improvements requires changing the system at academic medical centers. The critical pathway concept with its elements of team building, increased awareness of patient care issues, and education of all members of the healthcare team is an excellent tool for changing the system. Well suited to the postoperative management of complex procedures, critical pathways are one way of meeting the challenges of the oncoming "pay for performance" era.

\section{Acknowledgements}

The authors wish to acknowledge the Thomas Jefferson University Hospital nursing staff, anesthesia staff, and surgical house officers, who provided excellent care to the patients reported in this series. Additionally, the authors are grateful for the financial data provided by Jay Sial, Chief Operating Officer, JeffCARE, Inc. Jefferson Health System, and by Florence Williams, the Director of Clinical Operations in the Department of Surgery. 


\section{References}

1. Porter GA, Pisters PW, Mansyur C, Bisanz A, Reyna K, Stanford P, et al. Cost and utilization impact of a clinical pathway for patients undergoing pancreaticoduodenectomy. Ann Surg Oncol. 2000 Aug;7(7):484-9.

2. Pitt HA, Murray KP, Bowman HM, Coleman J, Gordon TA, Yeo CJ, et al. Clinical pathway implementation improves outcomes for complex biliary surgery. Surgery. 1999 Oct;126(4):751,6; discussion 756-8.

3. Wichmann MW, Roth M, Jauch KW, Bruns CJ. A prospective clinical feasibility study for multimodal "fast track" rehabilitation in elective pancreatic cancer surgery. Rozhl Chir. 2006 Apr;85(4):169-75.

4. Pritts TA, Nussbaum MS, Flesch LV, Fegelman EJ, Parikh AA, Fischer JE.

Implementation of a clinical pathway decreases length of stay and cost for bowel resection. Ann Surg. 1999 Nov;230(5):728-33.

5. Stephen AE, Berger DL. Shortened length of stay and hospital cost reduction with implementation of an accelerated clinical care pathway after elective colon resection. Surgery. 2003 Mar;133(3):277-82.

6. Cerfolio RJ, Bryant AS, Bass CS, Alexander JR, Bartolucci AA. Fast tracking after Ivor Lewis esophagogastrectomy. Chest. 2004 Oct;126(4):1187-94. 
7. Huber TS, Carlton LM, Harward TR, Russin MM, Phillips PT, Nalli BJ, et al. Impact of a clinical pathway for elective infrarenal aortic reconstructions. Ann Surg. 1998 May;227(5):691,9; discussion 699-701.

8. Leibman BD, Dillioglugil O, Abbas F, Tanli S, Kattan MW, Scardino PT. Impact of a clinical pathway for radical retropubic prostatectomy. Urology. 1998 Jul;52(1):94-9.

9. Markey DW, McGowan J, Hanks JB. The effect of clinical pathway implementation on total hospital costs for thyroidectomy and parathyroidectomy patients. Am Surg. 2000 Jun;66(6):533,8; discussion 538-9.

10. Kehlet H. Future perspectives and research initiatives in fast-track surgery. Langenbecks Arch Surg. 2006 Sep;391(5):495-8.

11. Campbell H, Hotchkiss R, Bradshaw N, Porteous M. Integrated care pathways. BMJ. 1998 Jan 10;316(7125):133-7.

12. Pearson SD, Kleefield SF, Soukop JR, Cook EF, Lee TH. Critical pathways intervention to reduce length of hospital stay. Am J Med. 2001 Feb 15;110(3):175-80.

13. Dy SM, Garg PP, Nyberg D, Dawson PB, Pronovost PJ, Morlock L, et al. Are critical pathways effective for reducing postoperative length of stay? Med Care. 2003 May;41(5):637-48.

14. Dy SM, Garg P, Nyberg D, Dawson PB, Pronovost PJ, Morlock L, et al. Critical pathway effectiveness: Assessing the impact of patient, hospital care, and pathway 
characteristics using qualitative comparative analysis. Health Serv Res. 2005 Apr;40(2):499-516.

15. Berenholtz S, Pronovost P, Lipsett P, Dawson P, Dorman T. Assessing the effectiveness of critical pathways on reducing resource utilization in the surgical intensive care unit. Intensive Care Med. 2001 Jun;27(6):1029-36.

16. Sohn TA, Yeo CJ, Cameron JL, Koniaris L, Kaushal S, Abrams RA, et al. Resected adenocarcinoma of the pancreas-616 patients: Results, outcomes, and prognostic indicators. J Gastrointest Surg. 2000 Nov-Dec;4(6):567-79. 
Table 1. Critical Pathway for Pancreaticoduodenectomy

\section{Day of Surgery:}

- Pre-operative heparin 5000 units subcutaneously

- TED stockings and sequential compression devices

- Perioperative antibiotics

- Central access per anesthesia assessment

- Nasogastric tube placed after induction of anesthesia

- Two JP drains (one each side)

- Night of surgery spent in ICU setting

- Intravenous PCA for analgesia

- Intravenous PPI

- Beta-blockade commenced orally preop or intravenously intraop

\section{Post-operative day \#1:}

- Remove nasogastric tube

- Discontinue antibiotics

- Start sips of water and ice chips $\leq 30 \mathrm{cc} / \mathrm{hr}$

- Out of bed ambulating

- Discontinue sequential compression devices, continue TEDs and heparin subcutaneously

- Continue intravenous beta-blockade and PPI

- Transfer to floor

Post-operative day \#2:

- Clear liquid diet

- Remove Foley catheter

- Minimize all IV fluids

- Begin diuresis and continue until discharge or patient reaches preoperative weight

- Continue TEDs, subcutaneous heparin, beta-blockade and PPI until hospital discharge

\section{Post-operative day \#3:}

- Regular diet with pancreatic enzymes

\section{Post-operative day \#4:}

- Switch all medications to oral route including analgesics

- Discontinue all IV fluids

- Remove JP drain with lowest volume (if appropriate)

\section{Post-operative day \#5:}

- Remove remaining JP drain (if appropriate)

- Distribute pre-printed discharge instructions

- Medical oncology and radiation oncology consults (if appropriate)

\section{Post-operative day \#6 or \#7:}

- Discharge home

- Arrange follow up appointment for 4 weeks after discharge

- Discharge medications: PPI, pancreatic enzymes, analgesics 
Table 2. Demographics and Intraoperative Parameters

$\mathrm{N}$

Surgical volume per month

Demographics

Age (years)

Mean \pm SE

Median

Sex

Female (\%)

Race

White (\%)

AA (\%)

Other (\%)

Pathology

Malignant (\%)

Operative blood loss $(\mathrm{ml}) \pm \mathrm{SE}$

Transfused PRBC (units) $\pm \mathrm{SE}$

Operative time (minutes) $\pm \mathrm{SE}$

Non-operative time in room (minutes) $\pm \mathrm{SE}$
Pre-pathway Post-pathway p-value

44

91

2.0

7.6
$63.9 \pm 1.3$

66

55

87

5

8

9

74

$$
549 \pm 48
$$

70

$646 \pm 29$

$0.72 \pm 0.16$

$0.64 \pm 0.13$

$435 \pm 14$

$379 \pm 12$

$76 \pm 2$

NS

NS

NS

NS

NS

NS

$95 \pm 4$
NS

NS

NS

$\mathrm{P} \leq 0.0001$

$\mathrm{P} \leq 0.0001$

Abbreviations: AA, African American; PRBC, packed red blood cells. 
Table 3. Postoperative Parameters

$$
\text { Pre-pathway Post-pathway P-value }
$$

Postoperative complications

Overall rate $(\%)$

Pancreatic fistula (\%)

Delayed gastric emptying (\%)

Wound infection (\%)

Other $(\%)$

Peri-operative mortality (\%)

Length of Stay (days; median)

Total hospital charges $\pm \mathrm{SE}$

Readmission within 30 days (\%)
44

9

7

9

25

2.3

13

$\$ 240,242 \pm \$ 32,490$

7
37

2

8

13

20

1.1

7

$\$ 126,566 \pm \$ 4883$

7.7
NS

NS

NS

NS

NS

NS

$\mathrm{P} \leq 0.0001$

$\mathrm{P} \leq 0.0001$

NS 